\title{
Surgical Treatment and Prognosis Analysis of Primary Carcinoma of Gallbladder
}

\author{
Guotao Fang ${ }^{1}$, Qi Zhang ${ }^{2, * *}$ \\ ${ }^{1}$ The Second Ward Department of Oncology, Affiliated Hospital of Hebei University, Baoding, P. R. China \\ ${ }^{2}$ Department of Clinical Medicine, Medical College of HeBei University, Baoding, P. R. China
}

\section{Email address:}

1377909325@qq.com (Qi Zhang)

${ }^{*}$ Corresponding author

\section{To cite this article:}

Guotao Fang, Qi Zhang. Surgical Treatment and Prognosis Analysis of Primary Carcinoma of Gallbladder Clinical Medicine Research. Vol. 6, No. 3, 2017, pp. 111-115. doi: 10.11648/j.cmr.20170603.19

Received: April 10, 2017; Accepted: April 24, 2017; Published: May 4, 2017

\begin{abstract}
To discuss the effects of different treatment methods on the prognosis of primary carcinoma of gallbladder (PCG) patients. The clinical statistics of 99 cases of PCG patients were retrospectively analyzed, and the prognosis multivariate analysis was performed. Results: $52 \%$ of the patients suffered from PCG and gallstones simultaneously, and 82.8\% (82/99) of the patients were diagnosed as PCG before surgeries. 86 cases of patients were subjected to routine exploratory surgeryies, and the radical resection rate was 33.72\% (29/86). The overall 5-year survival rate was $6.28 \%$, and the 1-year, 3-year and 5-year survival rates of the radical resection group were $78.43 \%, 48.49 \%$ and $23.77 \%$, respectively. The results were significantly different from those of the palliative surgery group, the exploratory laparotomy group and the non-surgical group. The multivariate analysis of Cox model reveals that surgical method was significantly correlated with the invasion depth of tumor and the prognosis of gallbladder carcinoma. The treatment efficacy of PCG might be improved by increasing the early diagnosis rate and performing radical resection which are accompanied by radiotherapy and chemotherapy.
\end{abstract}

Keywords: Gallbladder Tumor, Surgery, Prognostic Factor

\section{Introduction}

Gallbladder carcinoma commonly refers to the primary carcinoma of gallbladder (PCG). Histologically speaking, adenocarcinoma accounts for the highest proportion (more than $80 \%$ ), which is followed by squamous-cell carcinoma, mixed carcinoma and undifferentiated carcinoma [1]. The long-term treatment efficacy of gallbladder carcinoma is worse than those of liver cancer and pancreatic cancer due to its high degree of malignancy, easy early metastasis, difficult diagnosis, insensitive to chemotherapy drugs and etc. Gallbladder carcinoma is generally symptomatized as [2]: 1) digestive tract symptoms: Most patients (90\%) suffer from dyspepsia, aversion to greasy food, belching and poor appetite, which are attributed to the dysfunction of gallbladder that hinders the digestion of fatty substances. 2) Upper right abdominal pain: $80 \%$ of gallbladder carcinoma patients also suffer from ggallstones, the symptoms of which are similar to those of cholecystitis, including upper right abdominal discomfort and continuous secret anguish or dull pain, accompanied by paroxysmal sharp pain radiating to the right shoulder. 3) Upper right abdominal mass: The masses that can be found in the upper right abdomens or upper abdomens of approximately half of the patients are commonly enlarged gallbladders. 4) Icterus and pruritus: The symptoms often occur in advanced gallbladder carcinoma patients, which are induced by the invasion of bile duct by tumor tissues or biliary obstruction oppressed by metastasized swelling lymph nodes. Therefore, the bile that cannot flow into the intestinal tract flows back to blood, leading to the xanthochromia of skin mucosa, and skin itching especially in the night that cannot be mitigated. 5) Pyrexia and athrepsia: The pyrexia of approximately $25 \%$ of the patients mainly results from secondary infection of bilitary tract. The advanced patients are subject to athrepsia and even cachexia.

PCG stems from unknown reasons without particular symptoms and signs, leading to low early diagnosis rate and poor prognosis. Most PCG patients are not aware of the symptoms until the advanced stage, thereby resulting in low 
surgical resection rate and low 5-year survival rates ranging between $0 \%-9 \%$ [3]. Surgical should be given priority in the treatment of early-stage PCG. Doctors should endeavor to resect the gallbladder undergoing pathological changes after they get permission from PCG patients. An expanded dissection surgery is needed in case of lesion-involved gallbladder muscle layer, including the resection of liver tissues near gallbladder bed, soft tissues of hepatoduodenal ligament, and lymph nodes around gallbladder. Besides, the extrahepatic bile duct should also be resected if it is potentially involved [4].

In this study, the clinical data of 99 PCG patients enrolled in our hospital from 2002-2012 were retrospectively analyzed.

\section{Materials and Methods}

\subsection{General Information}

99 patients (M: 37 cases; F: 62 cases) aging between 36-81 years old (average: 57 years old; $>52$ years old: 75 cases) were selected. Accompanied gallstones with the history of 6 months to 32 years: 52 cases $(52.5 \%)$; recurrent biliary colic: 36 cases (36.3\%). Clinical signs: celiodynia ( 83 cases, $83.8 \%$ ), icterus (43 cases. 43.4\%), athrepsia (33 caes, 33.3\%) and abdominal mass (25 cases, $25.2 \%$ ).

\subsection{Radiography and Biochemical Examination}

Ultrasonic diagnosis

The ultrasonic diagnosis rate was $81.8 \%(81 / 99)$. The 18 cases that had not been diagnosed include 10 cases of gallstone accompanied chronic cholecystitis, 3 cases of hilar bile duct cancer, 2 cases of gallbladder adenomyomatosis, 2 cases of pancreatic head cancer, and 1 case of hilar space-occupying lesion.

CT diagnosis

The CT diagnosis rate was $89.6 \%(52 / 58)$. The 6 cases that had not been diagnosed include 1 case of chronic cholecystitis accompanied gallstone, 3 cases of hilar bile duct cancer and 2 cases of pancreatic head cancer.

MRI diagnosis

The MRI diagnosis rate was $78.5 \%(11 / 14)$, and the 3 cases that had not been diagnosed include 1 case of chronic cholecystitis accompanied gallstone and 2 cases of hilar bile duct cancer.

Encoscopic retrograde cholangio-pancreatography (ERCP). The ERCP [5] diagnosis rate was $13.3 \%(2 / 15)$.

Biochemical examination

The positive rates of serum CEA and CA-199 were $28.3 \%$ $(28 / 99)$ and 43.4\% (43/99), respectively.

\subsection{Pathological Data}

The 87 cases with completed pathological date include 71 cases of adenocarcinoma (81.6\%), 6 cases of papillary adenoma, 3 cases of tubular adenoma, 4 cases of squamous epithelial carcinoma, 4 cases of mucinous adenocarcinoma and 1 case of adenosquamous cell carcinoma. Histologically speaking, there were 24, 2621 and 13 cases of highly differentiated, mediumly differentiated, slightly differentiated and undifferentiated carcinomas.

\subsection{Clinical Staging and Treatment}

The Nevin grading and treatment methods of the 99 patients are shown in Table 1.13 out of the 99 patients did not undergo surgeries owing to poor systemic status, functional disorders of other vital organs and/or extensive metastasis, otherwise the patients were treated by surgeries with the radical resection rate of $33.72 \%$ (29/86). The radical resection group consists of 8 cases of gallbladder resection, 13 cases of standard radical resection and 8 cases of expanded radical resection (gallbladder resection + heptic segment resection + skeletonization of hepatoduodenal ligament: 3 cases; gallbladder resection + pancreaticoduodenectomy: 3 cases; gallbladder resection + right hemiliver resection: 2 cases). The palliative resection group consists of gross- or margin-positive patients. The exploratory laparotomy group were subjected to internal and external drainage, and/or biopsy. The non-surgical group were subjected to TCD drainage, interventional chemotherapy or intravenous chemotherapy, etc. None of the 86 patients died after surgeries, and the incidence of complications was $15.11 \%(13 / 86)$, including 1 case of bile leak, 1 case of incision infection, 1 case of subphrenic effusion, 3 cases of bile duct infection, 2 cases of abdominal infections, 2 cases of pleural effusion and 2 cases of liver failure.

Table 1. Nevin grading and treatment methods of the 99 patients.

\begin{tabular}{llllll}
\hline Nevin grading & Radical resection & Palliative surgery & Exploratory laparotomy and drainage & Non-surgical treatment & Total \\
\hline I & 6 & 0 & 0 & 0 & 6 \\
II & 7 & 4 & 0 & 0 & 2 \\
III & 9 & 2 & 1 & 3 & 11 \\
IV & 7 & 11 & 2 & 8 & 23 \\
V & 0 & 10 & 27 & 13 & 45 \\
Total & 29 & 27 & 30 & & 99 \\
\hline
\end{tabular}

\subsection{Follow-Up}

The 86 patients were followed up for 1 month to 5 years. The follow-up rate was $86.7 \%(86 / 99)$, and the failure rate of follow-up was $13.1 \%(13 / 99)$. The two groups did not differ significantly.

\subsection{Statistical Analysis}

Statistical analysis was performed using SPSS 13.0. The numeration data of each group were compared by the Chi 
square test, and the measurement data of each group were compared by the $t$ test. The single factor of survival prognosis was analyzed by the Kaplan-Meier method and the Log rank test. The single factors affecting prognosis significantly were further analyzed by the Cox multivariate method.

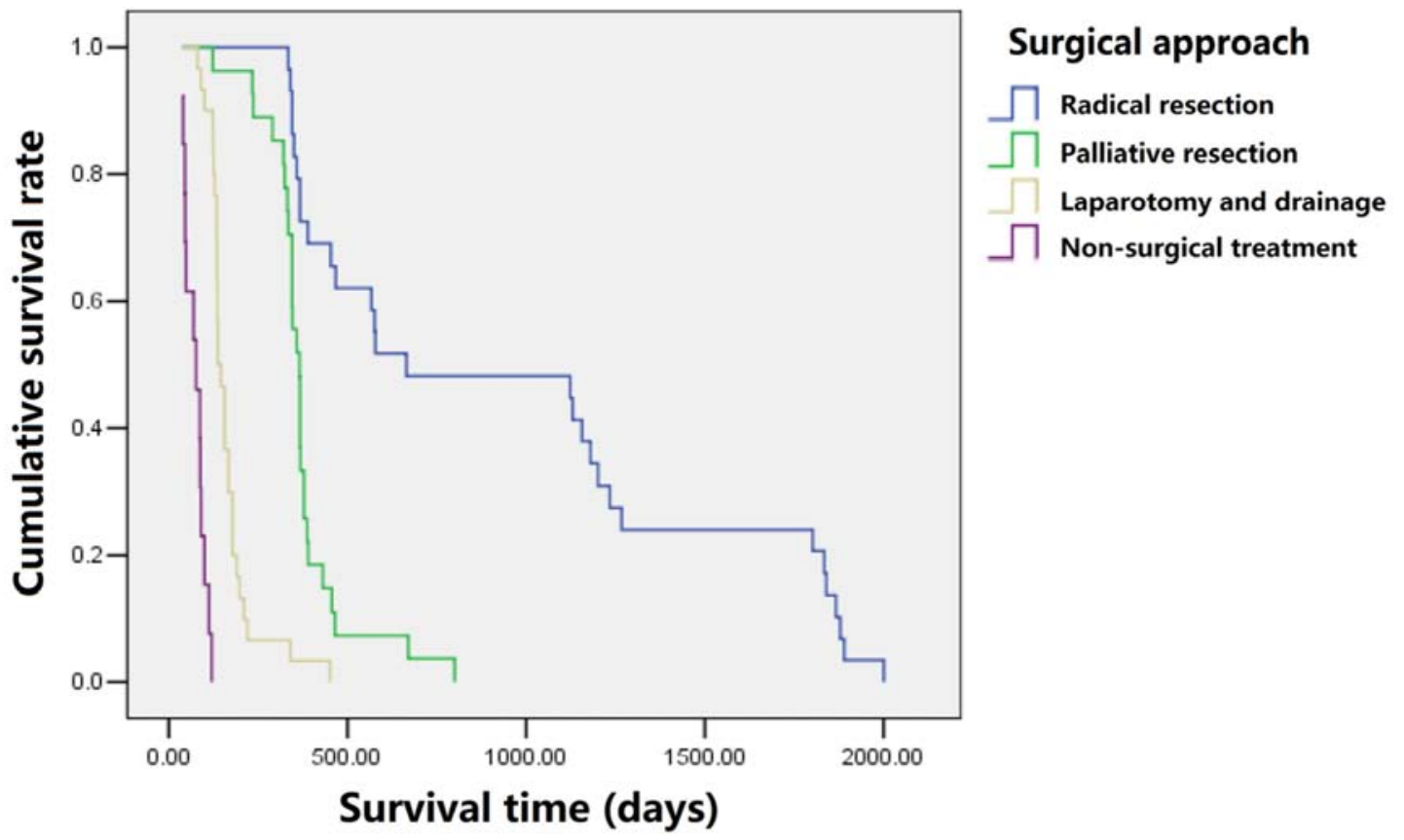

Figure 1. Survival rates of different treatment groups.

\section{Results}

\subsection{Different Treatment Methods and Survival Rates}

The overall 5-year survival rate was $6.28 \%$, and the 1-year, 3 -year and 5-year survival rates of the radical resection group were $78.43 \%, 48.49 \%$ and $23.77 \%$, respectively. The results were significantly different from those of the palliative surgery group, the exploratory laparotomy group and the non-surgical group $(\mathrm{P}=0.001,0,0)$, whereas the three groups did not differ significantly $(\mathrm{P}=0.063,0.085,0.941)$. The total surgical resection rate and the radical resection rate were $67.6 \%(67 / 99)$ and $33.72 \%(29 / 86)$, respectively. The Log-rank test reveals that there were significant overall differences between the 4 groups $(\chi 2=79.609, \mathrm{P}<0.001)$ (Table 2). The survival rate SPSS curves of different treatment methods are shown in Figure 1.
Table 2. Treatment methods and survival time of the 99 patients.

\begin{tabular}{lll}
\hline Treatment method & $\begin{array}{l}\text { Survival time } \\
\text { (median) }\end{array}$ & $\begin{array}{l}\text { Standard } \\
\text { error }\end{array}$ \\
\hline Radical resection & 686.0 & 34.970 \\
Palliative resection & 355.0 & 34.568 \\
Exploratory laparotomy and drainage & 136.0 & 19.879 \\
Non-surgical treatment & 78.0 & 2.367 \\
\hline
\end{tabular}

\subsection{Factors Influencing Prognosis}

The survival time of each group was counted by the Kplan-Meier method, which was further compared by the Log-rank method. The Cox model regression analysis (Table 3) shows that postoperative survival time was significantly correlated with surgical method and tumor invasion depth, but it was not significantly correlated with the other factors [6].

Table 3. Cox proportional hazard model single-factor analysis of gallbladder carcinoma.

\begin{tabular}{|c|c|c|c|c|c|c|c|c|}
\hline \multirow{2}{*}{ Variable } & \multirow{2}{*}{ Regression coefficient } & \multirow{2}{*}{$\begin{array}{l}\text { Standard } \\
\text { error }\end{array}$} & \multirow{2}{*}{$\begin{array}{l}\text { Standard } \\
\text { deviation }\end{array}$} & \multirow{2}{*}{$\begin{array}{l}\text { Degree of } \\
\text { freedom }\end{array}$} & \multirow{2}{*}{ P value } & \multirow{2}{*}{$\begin{array}{l}\text { Relative } \\
\text { risk }\end{array}$} & \multicolumn{2}{|c|}{$95.0 \%$ CI for $\operatorname{Exp}(B)$} \\
\hline & & & & & & & Lower & Upper \\
\hline Gender & 0.438 & 0.347 & 1.590 & 1 & 0.210 & 1.553 & 0.778 & 3.101 \\
\hline Age & 0.671 & 0.360 & 3.535 & 1 & 0.059 & 1.950 & 0.976 & 3.914 \\
\hline Preoperative serum bilirubin & -0.015 & 0.012 & 1.595 & 1 & 0.211 & 0.983 & 0.957 & 2.082 \\
\hline Oncological cell grading & -0.137 & 0.444 & 0.109 & 1 & 0.748 & 0.865 & 0.361 & 11.938 \\
\hline Tumor position & 0.805 & 0.853 & 0.890 & 1 & 0.343 & 2.238 & 0.422 & 7.808 \\
\hline Distant metastasis & 0.861 & 0.612 & 1.983 & 1 & 0.158 & 2.360 & 0.713 & 1.321 \\
\hline Histological pathology type & 0.031 & 0.095 & 1.082 & 1 & 0.300 & 1.101 & 0.916 & 1.277 \\
\hline Accompanied by gallstone & -0.132 & 0.114 & 0.093 & 1 & 0.755 & 1.032 & 0.831 & 1.401 \\
\hline Nevin grading & -0.202 & 0.247 & 0.315 & 1 & 0.571 & 0.871 & 0.552 & 1.210 \\
\hline Surgical method & 0.613 & 0.203 & 1.044 & 1 & 0.306 & 1.843 & 1.242 & 2.744 \\
\hline Tumor invasion depth & 0.575 & 0.249 & 9.178 & 1 & 0.019 & 1.780 & 1.093 & 2.895 \\
\hline
\end{tabular}




\section{Discussion}

\subsection{Diagnosis}

The early-stage gallbladder carcinoma can hardly be diagnosed [7]. The early diagnosis rate can be increased by radiologically following up the high-risk population regularly [8]. Although type B ultrasonic is commonly utilized to screen gallbladder carcinoma, it is prone to the interference of obesity and gastrointestinal gas. In contrast, gallbladder is scanned by endoscopic ultrasound (EUS) probe only through gaster and duodenal, which avoids the influence of gas in the gastrointestinal tract, contributes to the early diagnosis of gallbladder carcinoma, and determines the degree of invasion [9]. Color Dopple ultrasound distinguishes gallbladder carcinoma from intragallbladder cholestasis according to the blood flow signal. A high-speed blood flow indicates gallbladder carcinoma with the sensitivity of $83.9 \%$ and the specificity of $80.5 \%$ [10]. CT functions in the diagnosis of gallbladder carcinoma in the distinguishing of it from chronic cholecystitis and gallbladder polyps, the clinical staging and the judgment about resectability. However, it is difficult to distinguish the postoperative local residues or recurrent cancer from surgical scars by CT [11]. Although MRI does not substantially help the early diagnosis of gallbladder carcinoma, magnetic resonance cholangiopancreatography (MR-CP) is a non-invasive approach that may replace ERCP and PTC in the near future. CA19-9 and CEA can hardly be used to early diagnose gallbladder carcinoma.

\subsection{Treatment}

Surgery should be given priority in the treatment of gallbladder carcinoma, and different surgical methods should be selected according to the Nevin grading and individual differences. The surgical methods of gallbladder carcinoma include: cholecystectomy, expanded cholecystectomy (cholecystectomy + lymph node dissection), radical surgery of gallbladder carcinoma (cholecystectomy + lymph node dissection + wedge resection of the gallbladder bed), and expanded radical surgery of gallbladder carcinoma (basic radical surgery of gallbladder carcinoma + expanded hepatectomy + resection of adjacent involved organs + biliary tract reconstruction). Generally, the early stage (Nevin I and II) gallbladder carcinoma patients are merely in need of cholecystectomy The advanced stage (Nevin III and IV) patients should be subjected to radical or palliative surgeries according to the status per se. In this study, the 1-, 3- and 5 -year survival rates of the radical surgery group were apparently higher than those of the palliative surgery group. Therefore, basic or expanded radical surgeries are preferred. Todoroki et al. [12] reported that the 5-year survival rate of the radical surgery group was $35.5 \%$, and 24 patients even survived for more than 5 years. The 1-year survival rate of the expanded radical surgery group (maximum: 965 days) was $67.1 \%$, but all the patients who did not receive resection surgeries died within only 1 year. Thus, an expanded surgery can increase the radical cure rate and prolong the survival time as well. Nevertheless, radical resection may lead to complications more commonly. Meanwhile, the survival rate of the margin-positive patients did not differ from that of the gross-positive patients after radical resection [13]. On the other hand, the multivariate analysis reveals that lymph node metastasis and distant metastasis were not significantly correlated with prognosis, suggesting that expanded radical surgery targeting negative margin will necessarily increase the odds of perioperative death and complications. Therefore, the early diagnosis and the auxiliary treatment should be focused instead. It has been previously reported that [14] the survival rate of the patients who underwent radical surgeries and radiotherapy or chemotherapy synergetically was obviously higher than that of the patients who only received surgeries. Particularly, the 5-year survival rate of the patients with tumor residues could be substantially increased by radiotherapy. In summary, gallbladder carcinoma may result from mucous membrane epithelial metaplasia, dysplasia, mutation, bile evacuation disorder and carcinogenic substances (e.g. lithocholic acid, cholanthrene and methylcholanthrene) induced by chronic ischemia of gallbladder mucous membrane, recurrent injury and chronic inflammation in the presence of the suppression and stimulation of gallstones. As a result, a preventive resection of gallbladder is recommended for the high-risk patients in case of deterioration.

\section{References}

[1] Cho SY, Han SS, et al. T-category reflects the histopathologic characteristics of gallbladder carcinoma [J]. Eur J Surg Oncol, 2012, 38 (6): 537-42.

[2] Ghosh M, Bhattacharyya NK, Ghoshsengupta S. Carcinoid tumour of gall bladder--a case report [J]. J Indian Med Assoc, 2011, 109 (3): 198-9.

[3] Kim K, Chie EK,et al. Postoperative chemoradiotherapy for gallbladder carcinoma [J].Strahlenther Onkol, 2012, 188 (5): 388-92.

[4] Pais-Costa SR, Farah JF, et al. Gallbladder adenocarcinoma: evaluation of the prognostic factors in 100 resectable cases in Brazil [J]. Arq Bras Cir Dig, 2012, 25 (1)13-9.

[5] Kim KH, Kim TN. Etiology, clinical features, and endoscopic management of hemobilia: a retrospective analysis of 37 cases [J]. Korean J Gastroenterol, 2012， 59 (4) 296-302.

[6] Yao X, Zhou L, Han S, Chen Y. High expression of CXCR4 and CXCR7 predicts poor survival in gallbladder carcinoma [J]. J Int Med Res, 2011, 39 (4): 1253-64.

[7] Wakai T, Ajioka Y,et al. Morphological features of early gallbladder carcinoma [J]. Hepatogastroenterology, 2012, 59 (116): 1013-7.

[8] Giang TH, Ngoc TT, Hassell LA. Carcinoma involving the gallbladder: a retrospective review of 23 cases - pitfalls in diagnosis of gallbladder carcinoma [J]. Diagn Pathol, 2012, 27 (7): 10-11. 
[9] Hijioka S, Ikari T, et al. US - IDUS - EUS for diagnosis of biliary tract neoplasms [J]. Nihon Rinsho, 2006, 64 (1): 393-7.

[10] Konstantinidis IT, Bajpai S,et al. Gallbladder lesions identified on ultrasound. Lessons from the last 10 years [J]. J Gastrointest Surg, 2012, 16 (3): 549-53.

[11] Kumar R, Sharma P, et al. Role of 18F-FDG PET/CT in detecting recurrent gallbladder carcinoma [J]. Clin Nucl Med, 2012, 37 (5): 431-5.
[12] Todoroki T, Takahashi $\mathrm{H}$ et al. Outcomes of aggressive treatment of stage IV gallbladder carcinoma and predictors of survival [J]. Hepatogastroenterology, 1999, 46 (28): 2114-21.

[13] Choi SB, Han HJ, et al. Fourteen year surgical experience of gallbladder carcinoma: validity of curative resection affecting survival [J]. Hepatogastroenterology, 2012, 59 (113): 36-41.

[14] Wang SJ, Lemieux A, et al. Nomogram for predicting the benefit of adjuvant chemoradiotherapy for resectedgallbladder carcinoma [J]. J Clin Oncol, 2011, 29 (35): 4627-32. 